\title{
Criticality of Low-Energy Protons in Single-Event Effects Testing of Highly-Scaled Technologies
}

J. A. Pellish ${ }^{1}$, P. W. Marshall2, K. P. Rodbell ${ }^{3}$, M. S. Gordon ${ }^{3}$, K. A. LaBel ${ }^{1}$, J. R. Schwank ${ }^{4}$, N. A. Dodds ${ }^{4}$, C. M. Castaneda', M. D. Berg ${ }^{6}$, H. S. Kim ${ }^{6}$, A. M. Phan ${ }^{6}$, and C. M. Seidleck ${ }^{6}$

1: NASA Goddard Space Flight Center, Greenbelt, MD 20771 USA

2: NASA consultant, Brookneal, VA USA

3: IBM Thomas J. Watson Research Center, Yorktown Heights, NY USA

4: Sandia National Laboratories, Albuquerque, NM USA

5: University of California at Davis, Davis, CA USA

6: ASRC Federal Space \& Defense, Greenbelt, MD USA

Sandia is a multi-program laboratory operated by Sandia Corporation, a Lockheed Martin Company, for the United States

Department of Energy's National Nuclear Security Administration under Contract DE-AC04-94AL85000. 


\section{Acronyms}

- CMOS: complementary metal oxide semiconductor

- CNL: Crocker Nuclear Laboratory

- CSDA: continuous slowing down approximation

- DBU: double-bit upset

- DUT: device under test

- IBM YKT: Yorktown Heights, NY

- ICRU: International Commission on Radiation Units \& Measurements

- IEEE: Institute of Electrical and Electronics Engineers

- IUCF: Indiana University Cyclotron Facility

- LBNL: Lawrence Berkeley National Laboratory

- LEP: low-energy proton

- MCU: multi-cell upset (errors not necessarily in the same data word)

- Different from multi-bit upset (MBU)
- NIST: National Institute of Standards and Technology

- ASTAR and PSTAR are NIST tools, not acronyms

- NPTC: Northeast Proton Therapy Center

- SBU: single-bit upset

- SEEM: secondary electron emission monitor

- SEU: single-event upset

- SOI: silicon on insulator

- SRAM: static random access memory

- SRIM: Stopping and Range of lons in Matter (software program)

- TNS: Transactions on Nuclear Science

- TRIUMF: not an acronym - formerly the Tri-University Meson Facility, Vancouver, Canada

- UC Davis: University of California at Davis 


\section{Outline}

- Introduction

- Low-energy protons (LEPs)

- Test facility setup

- Protons vs. alphas

- DUT is $32 \mathrm{~nm}$ SOI CMOS $128 \mathrm{Mb}$ SRAM

- Heavy ion data baseline

- DUT is in a flip-chip package

- SBU and MCU SRAM data

- Die thickness reverse engineering with SRIM

- Summary

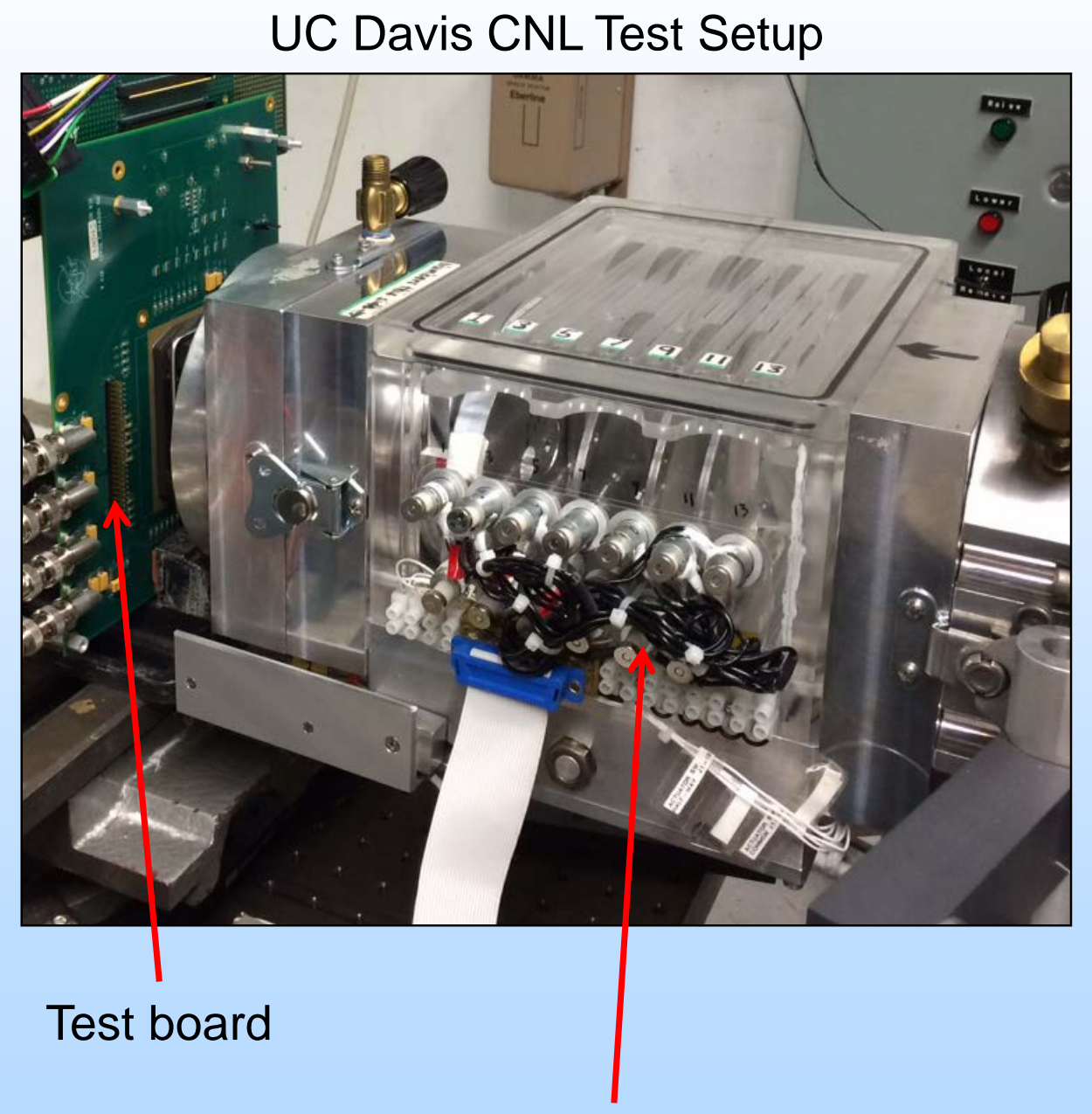

User-Controlled Degrader Foil Chamber 


\section{Early Low-Energy Proton Data}

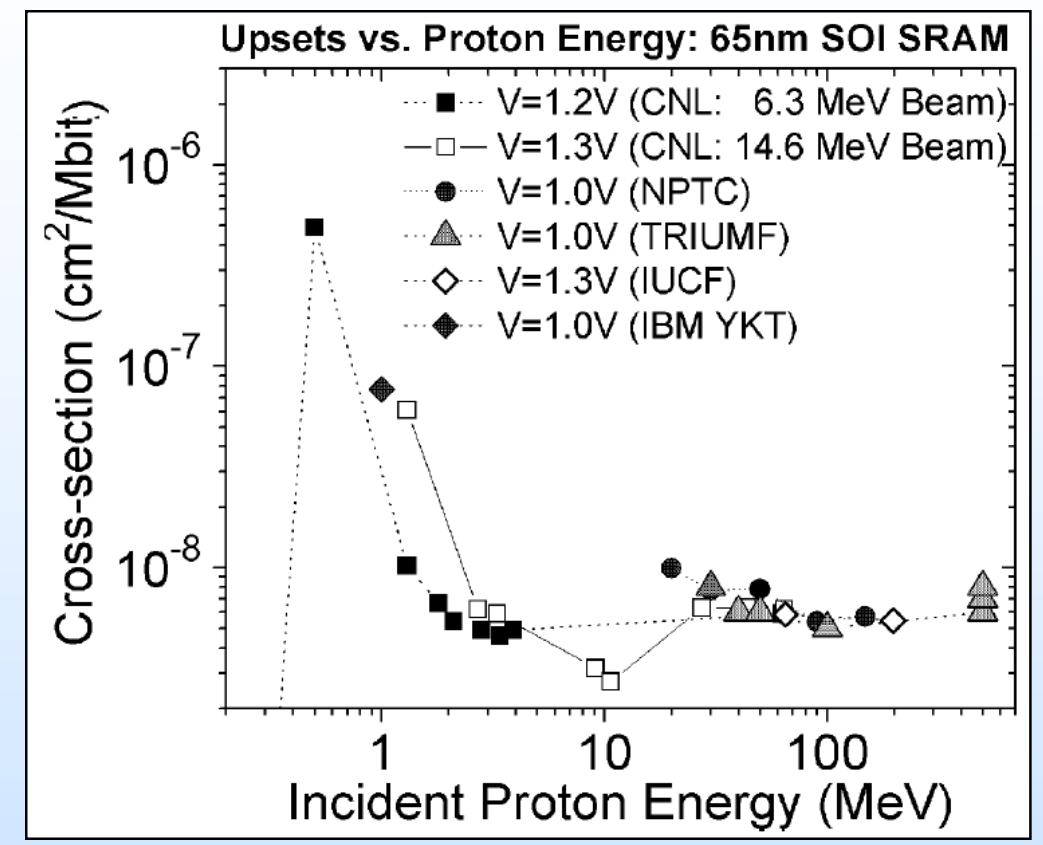

D. F. Heidel et al., IEEE TNS, vol. 6, 2008.

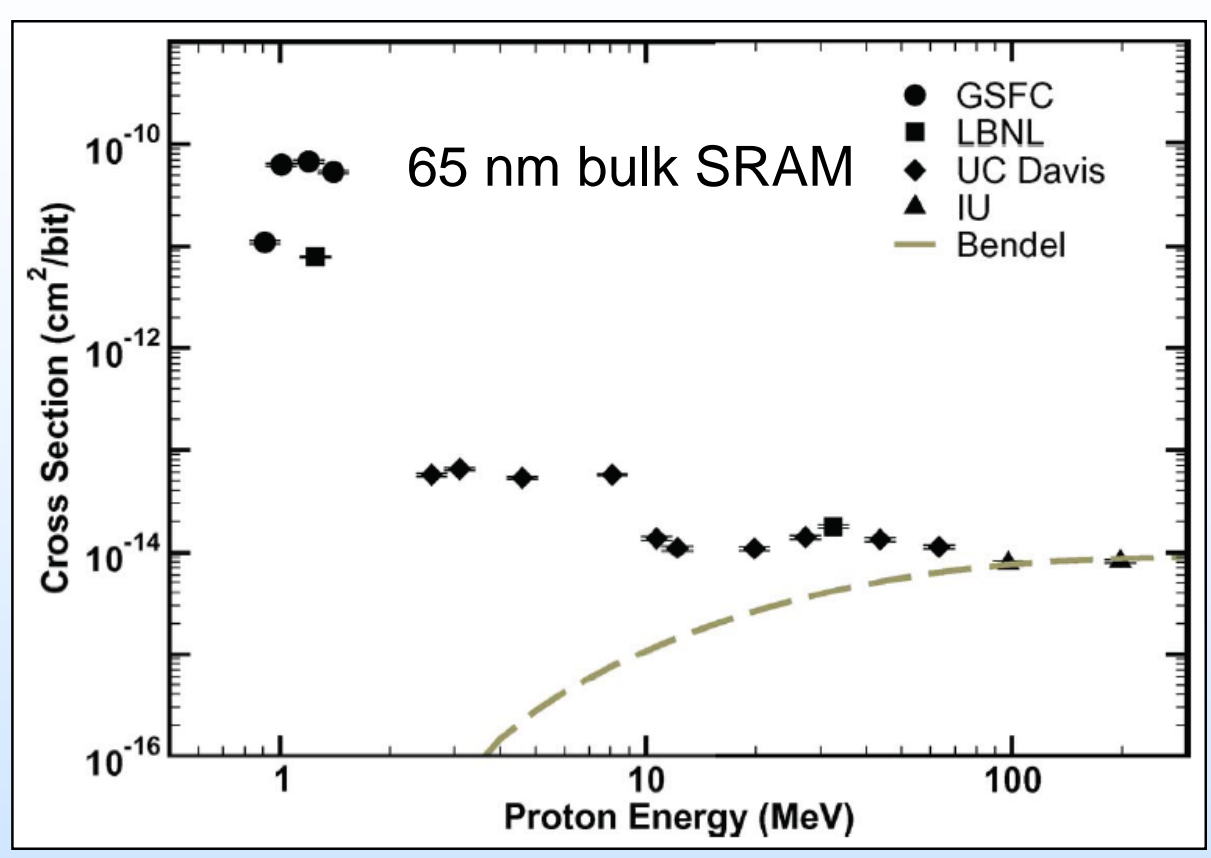

B. D. Sierawski et al., IEEE TNS, vol. 6, 2009.

- Cross sections are plotted as a function of incident proton energy inversely proportional to degrader thickness.

- Several implications: changes to the energy distribution shape, flux depletion near end-of-range, etc.

- Can plot data as a function of degrader thickness.

- How do we know the mean energy and standard deviation? 


\section{The Problem with LEPs - Range \& dEldx}

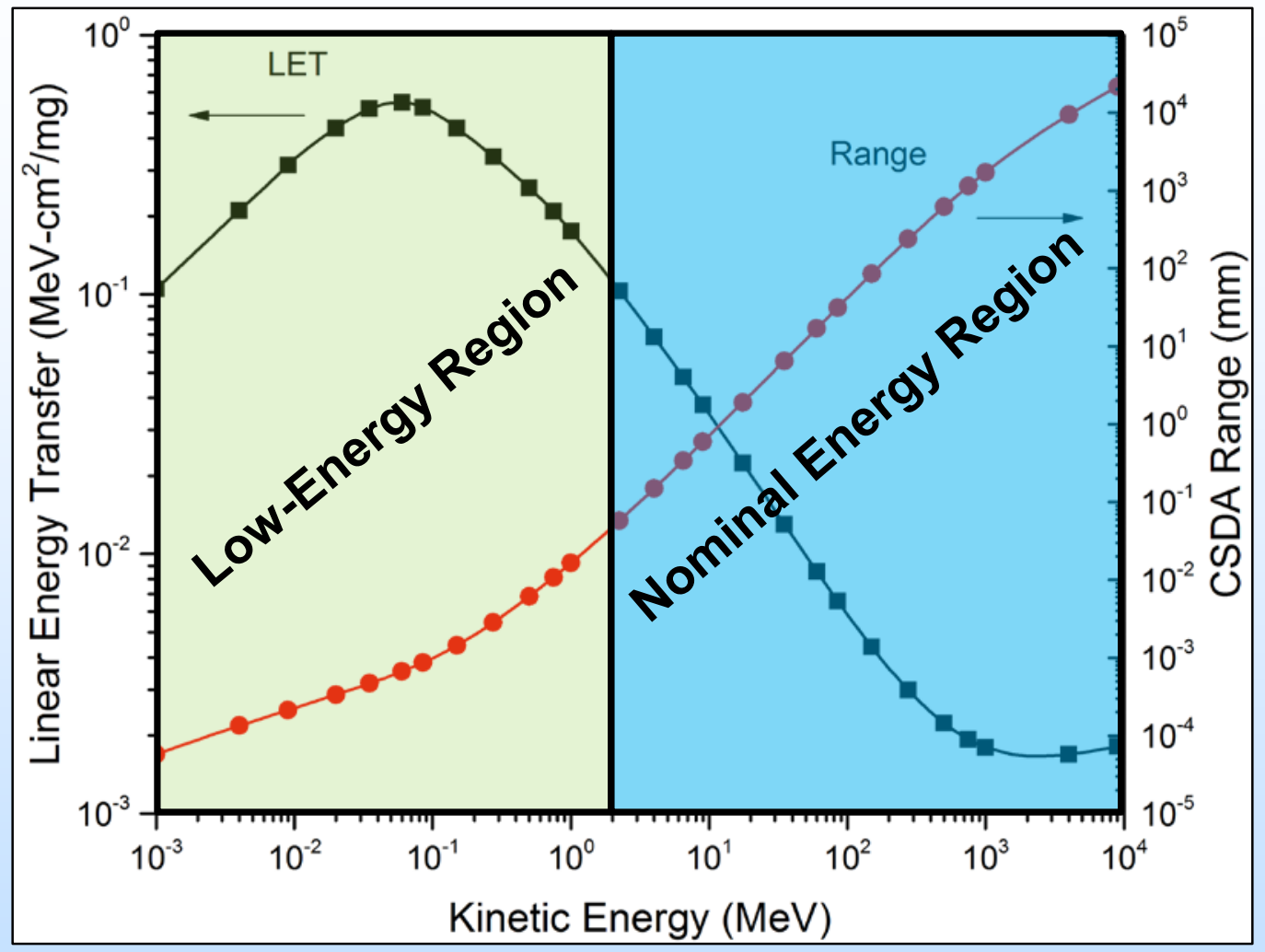

NIST PSTAR tool (ICRU Report 49, 1993).

- Greatest effect in the shortest distance

- Short range in region of interest implies flux depletion

- Cross sections tend to be uncorrected for flux loss and plotted as a function of known quantities (e.g., degrader thickness) 


\section{The Alpha $\left({ }^{4} \mathrm{He}\right)$ Alternative}

$\mathrm{H}^{+}$starts at $6.5 \mathrm{MeV}$

- CNL uses

$\sim 13 \mathrm{MeV} \mathrm{H}_{2}^{+}$to generate LEPS

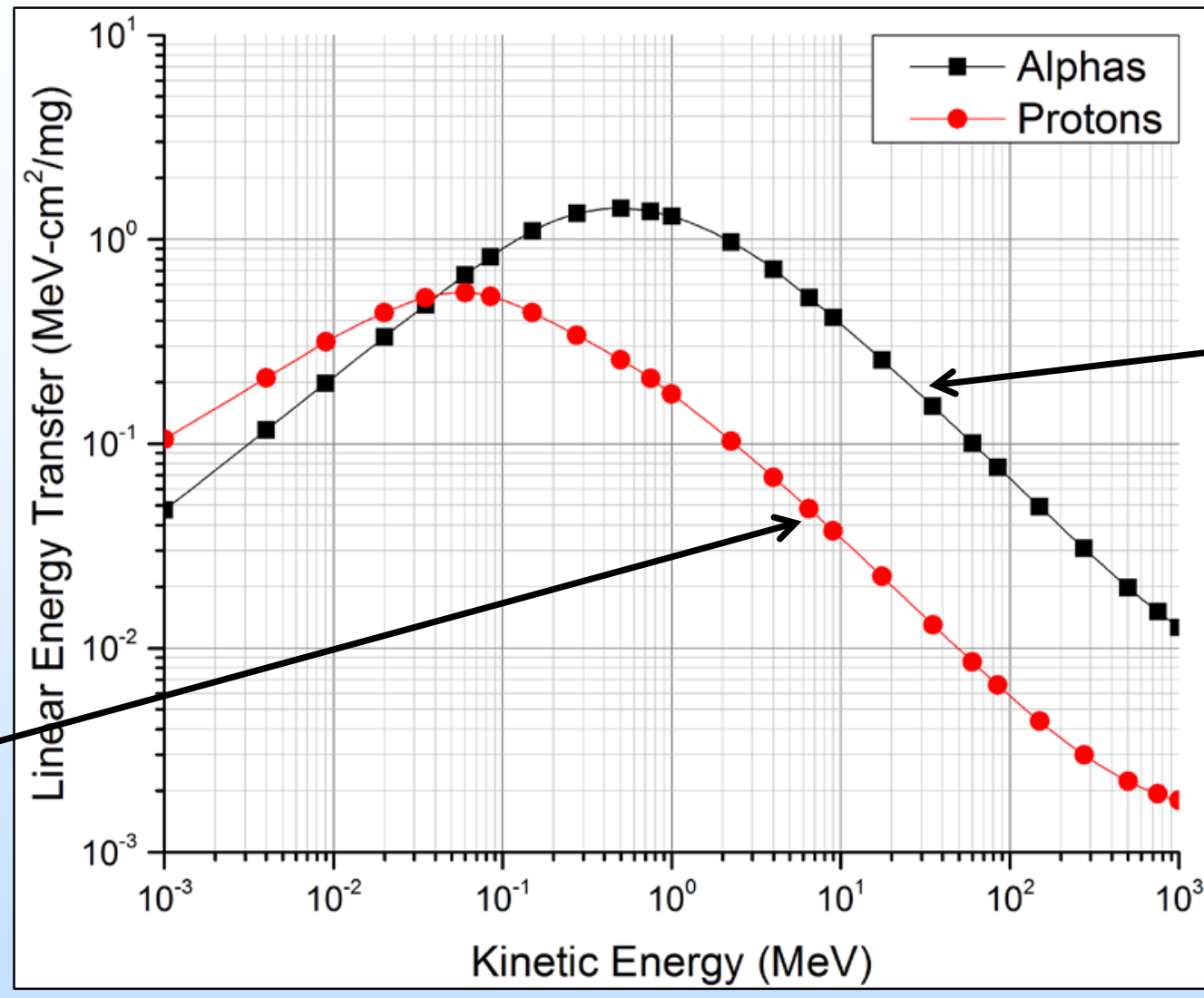

${ }^{4} \mathrm{He}$ starts at $30 \mathrm{MeV}$

NIST ASTAR/PSTAR tool (ICRU Report 49, 1993).

- Hypothesis: Alpha particles can replace LEPs for direct ionization single-event effects testing.

In the context of LEPs, mentioned as early as 2009 - B. D. Sierawski et al., including J. Pellish. 


\section{UC Davis Crocker Nuclear Laboratory}

Assuming Setup In-Air (can do vacuum)

- Beam diameter on 0.25 mil Ta foil is $\sim 5 / 16$ in.

- Defining collimator is 2.75 in with acceptance angle of $0.018 \mathrm{rad}$.

- Secondary electron emission monitor (SEEM) uses three 0.25 mil Al foils.

- User-selected degraders can be Al or Mylar.

- Exit window is $\mathbf{5}$ mil Kapton.

- Air gap is user-selected within experimental parameters.

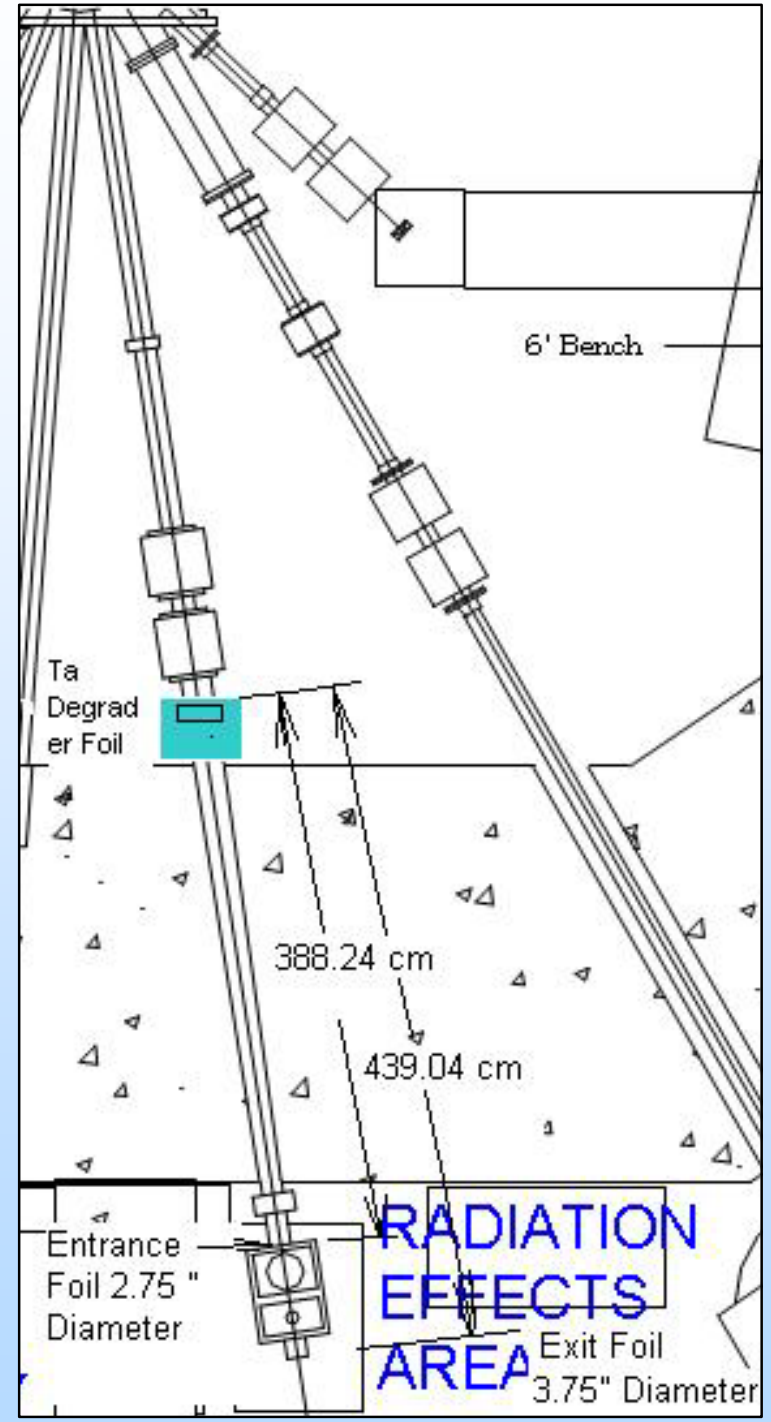

Courtesy of T. Essert and M. Van de Water (UCD/CNL). 


\section{$30 \mathrm{MeV}$ Alpha SEU Data 0x0000 Pattern}

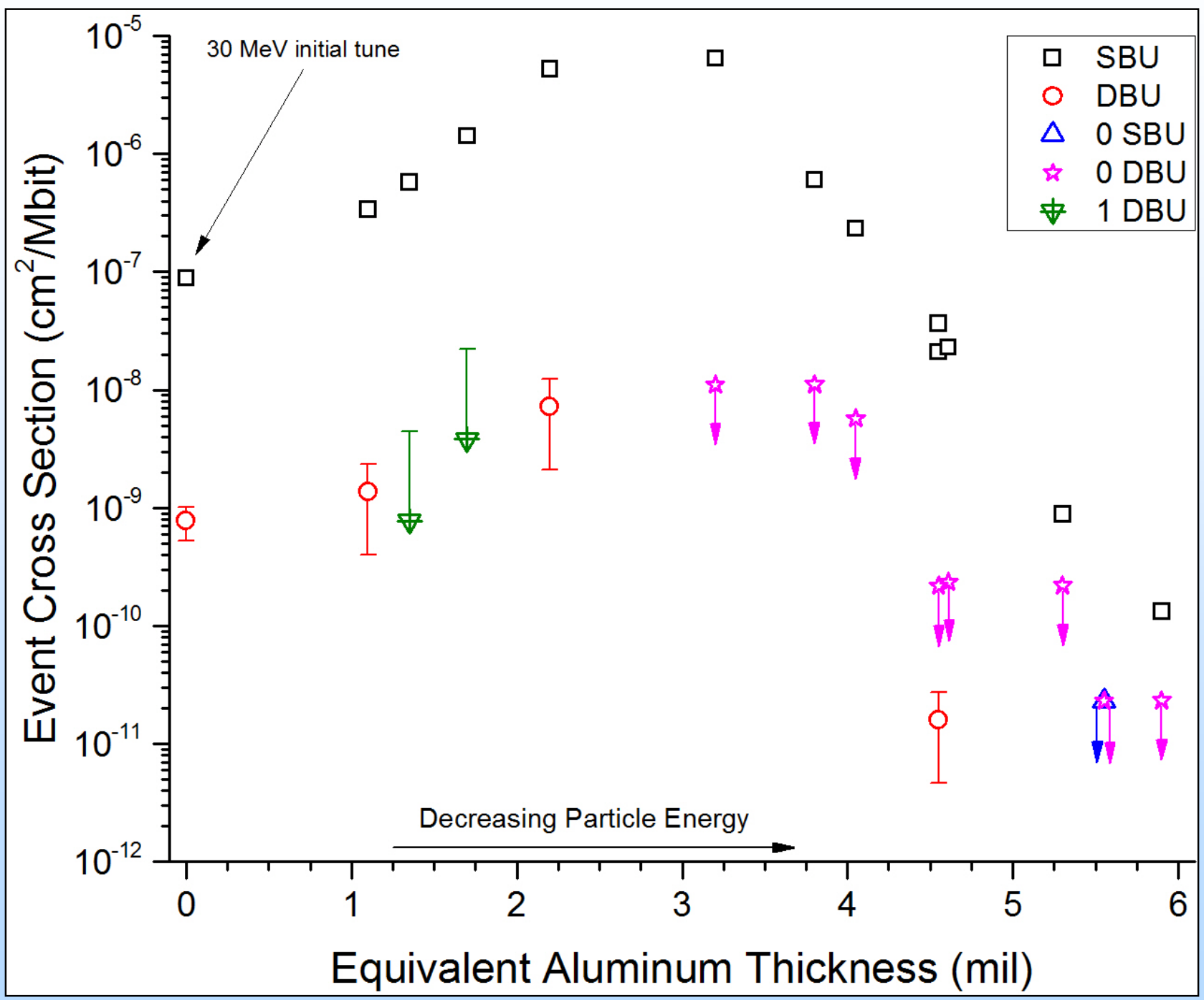

Error bars, if shown, are at the $90 \%$ confidence level. 


\subsection{MeV Proton SEU Data 0x0000 Pattern}

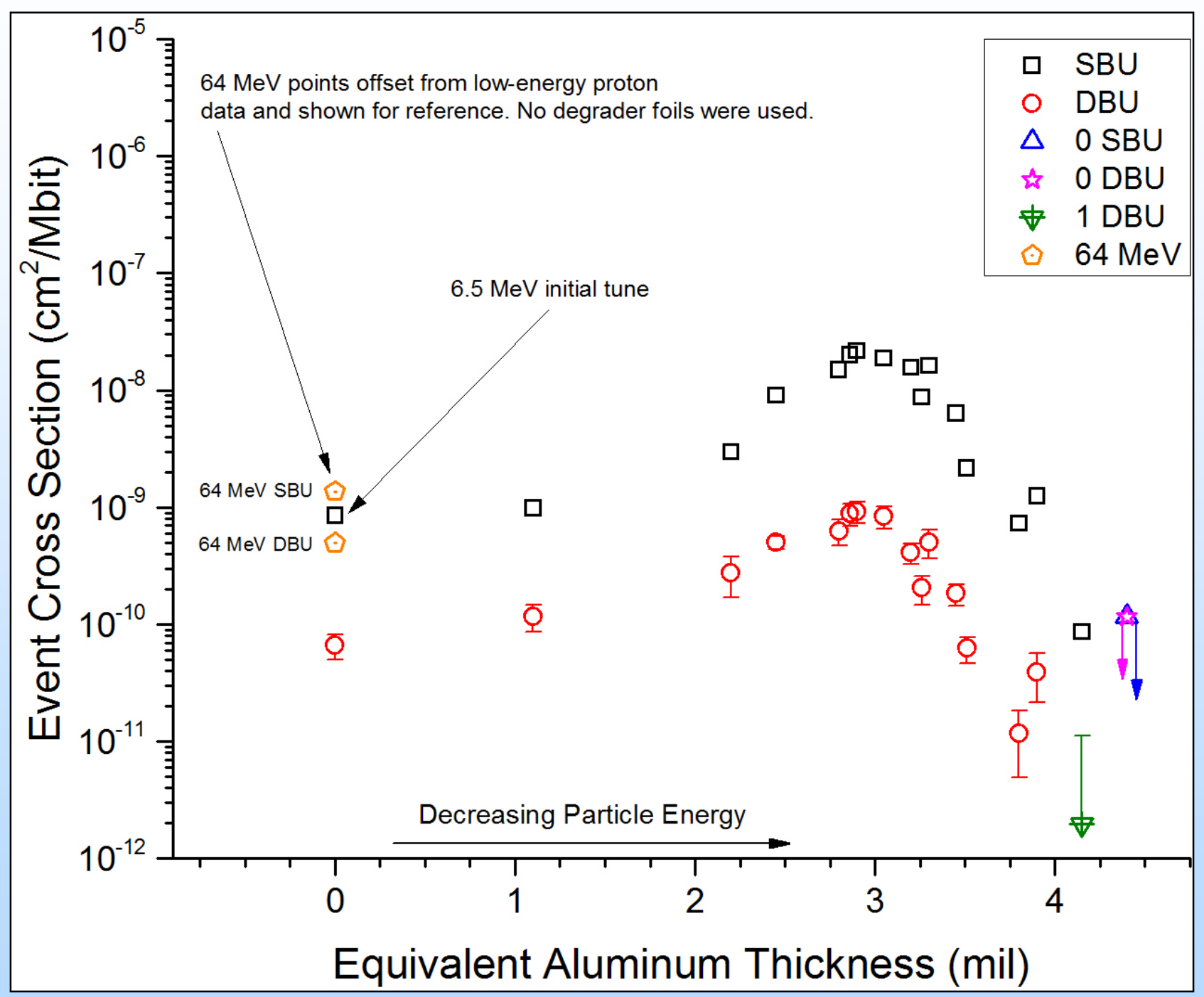

Error bars, if shown, are at the $90 \%$ confidence level. 


\section{Data Comparison - Side-by-Side}

Alpha

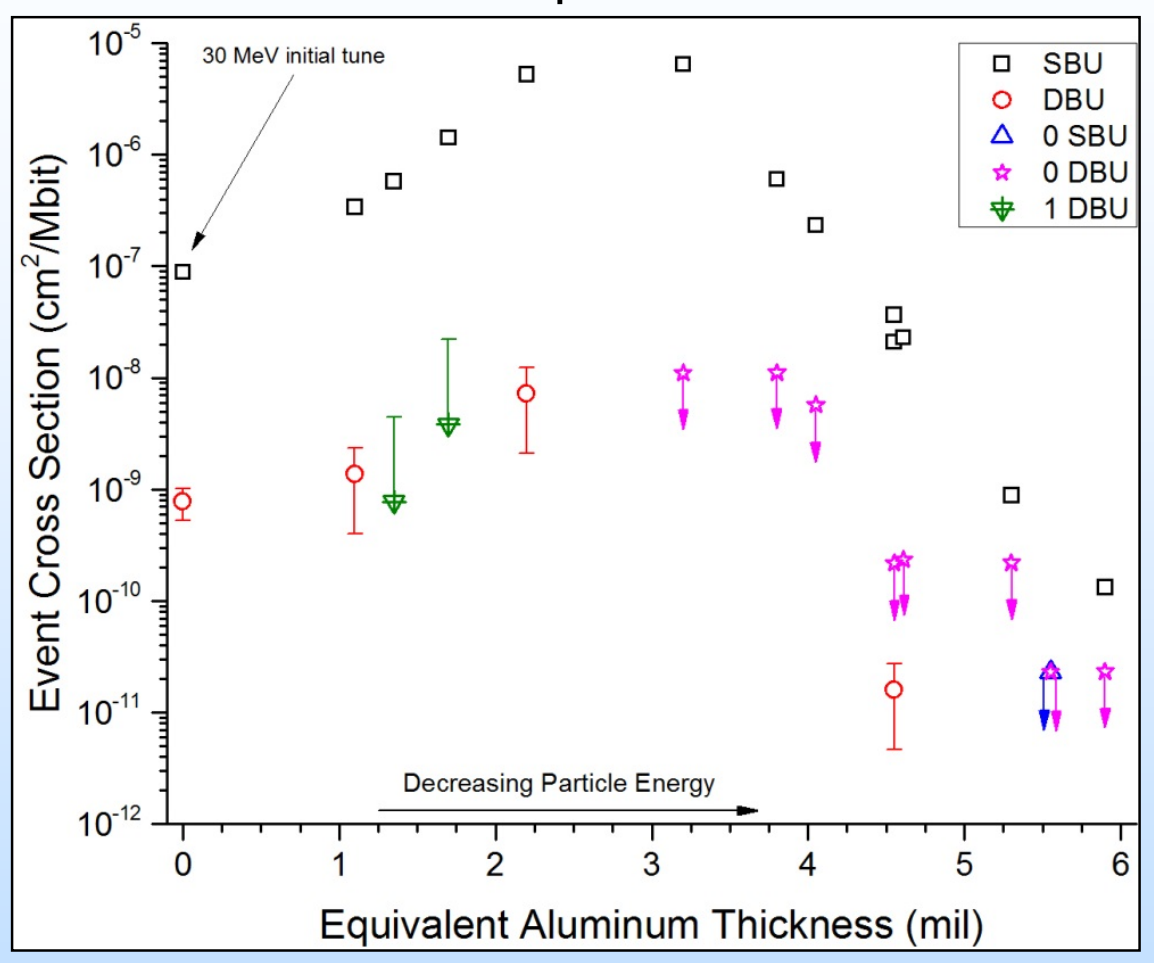

Proton

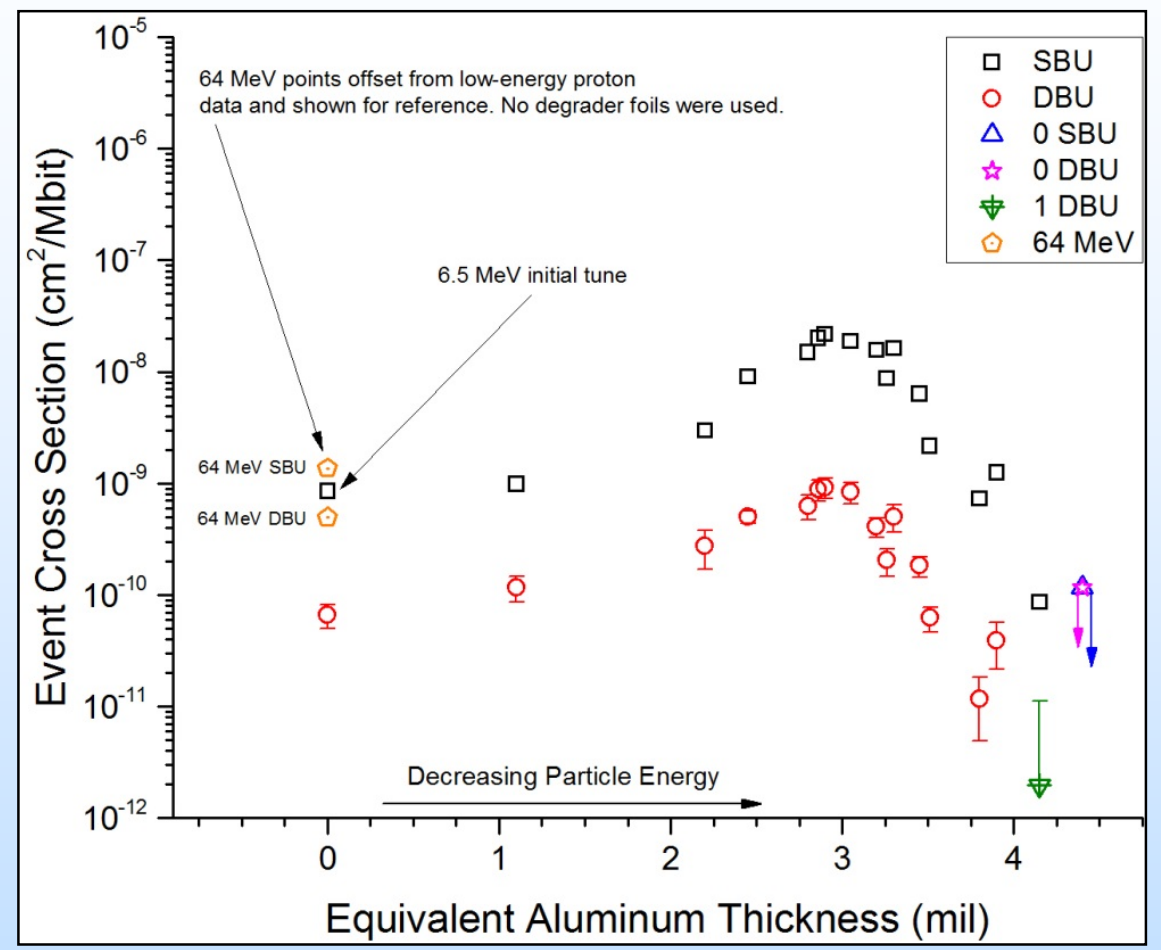

- On the low-energy side of the Bragg peak, the cross sections are similar, but the type of events are not.

- One of the key features is the separation between SBUs and DBUs. 


\section{Alpha \& Proton DBU Fraction}

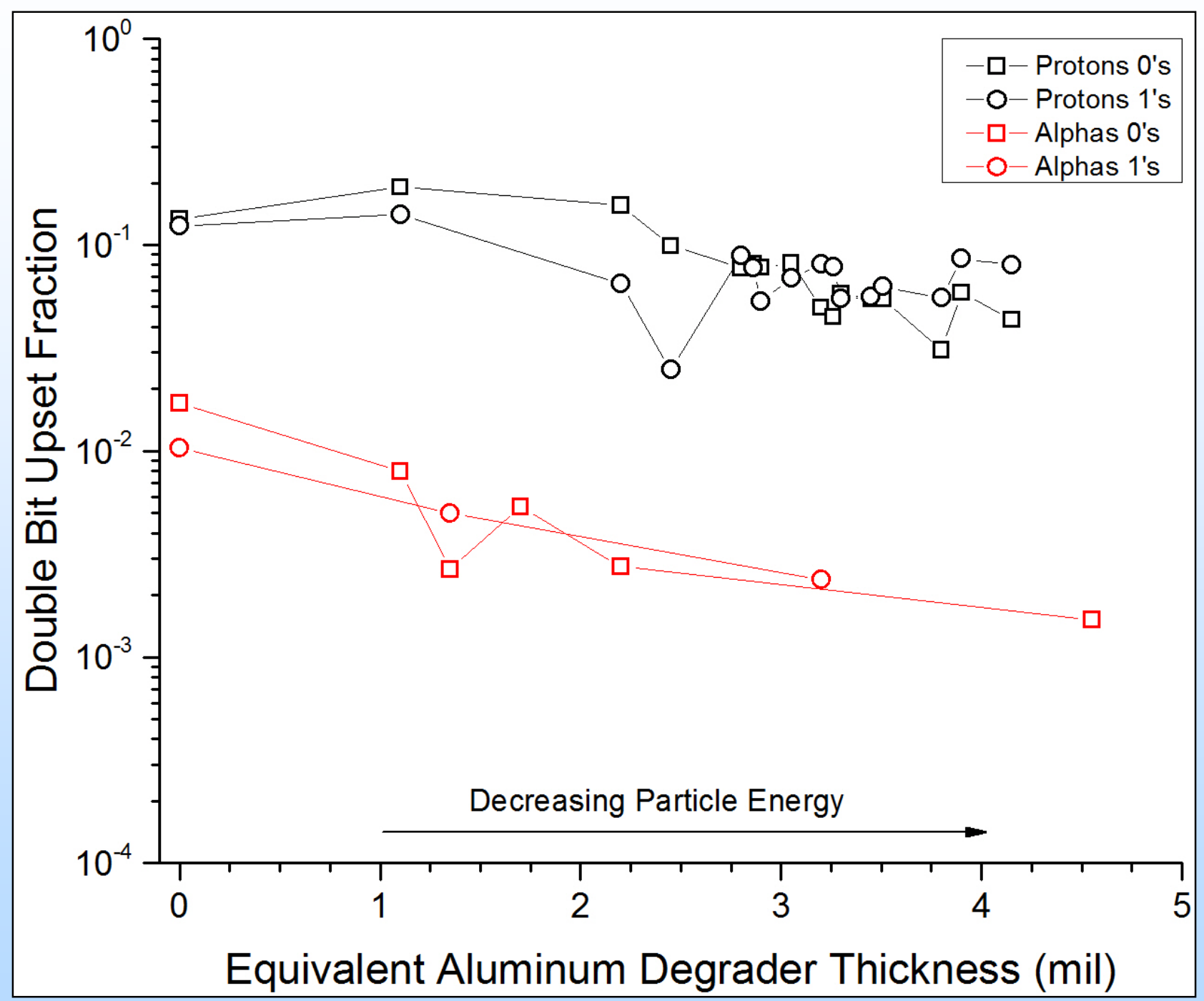

Shows both 0x0000 and 0xFFFF data patterns. 


\section{Physical Failure Maps - Alphas 0x0000 Pattern}

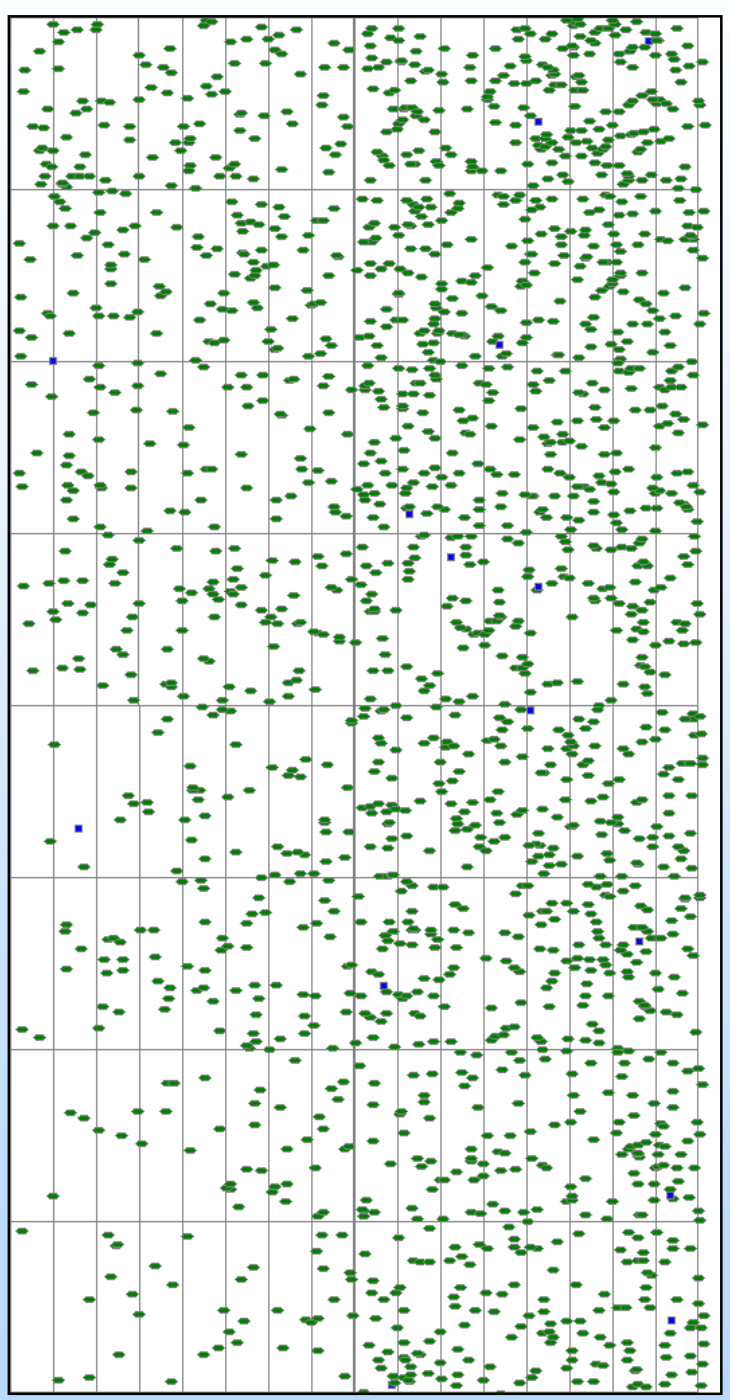

- $30 \mathrm{MeV}$ primary

- No user degrader

- Actual alpha energy below primary tune

- Only 14 total MCUs

- All DBUs

- 4 on left, 10 on right

Single

WordLine

Bitl ine

Diagonal

- For alpha/proton comparison, remember, these plots are absolute event counts.

- Total fluence and LET dependencies.

In legend $-\mathrm{MBU}=\mathrm{MCU}$ 


\section{Physical Failure Maps - Protons 0x0000 Pattern}

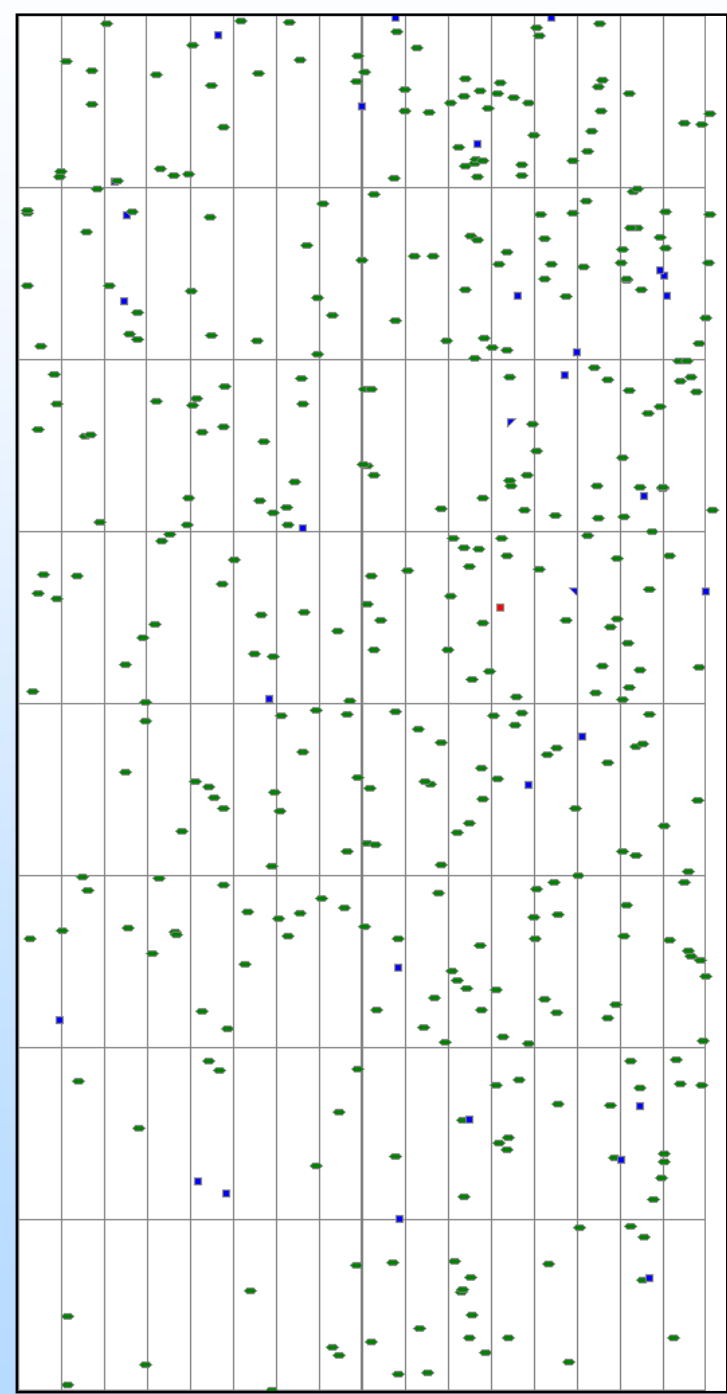

- 6.5 MeV primary

- No user degrader

- Actual proton energy below primary tune

- 32 total MCUs

- MBU2

$\nabla_{\text {MBU3 }}$

$\checkmark$ MBU4

Single

WordLine

BitL ine

Diagonal

- 13 on left, 19 on right

- Most MCUs are word-line DBUs

- One 3-bit MCU and one 4-bit MCU - both word line

- For alpha/proton comparison, remember, these plots are absolute event counts.

- Total fluence and LET dependencies.

In legend $-\mathrm{MBU}=\mathrm{MCU}$ 


\section{Incorporation of SRIM Simulations to Calculate Die Thickness}

- Fail mapping can be used to isolate areas of interest for analysis.

- Beam stopping degrader thickness can be used to "back out" silicon die thickness.

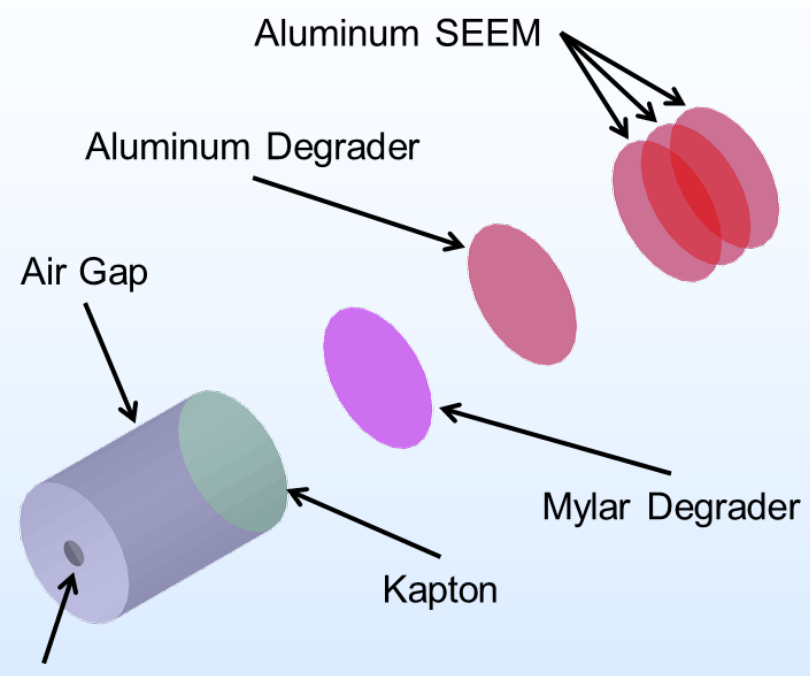

Silicon Target
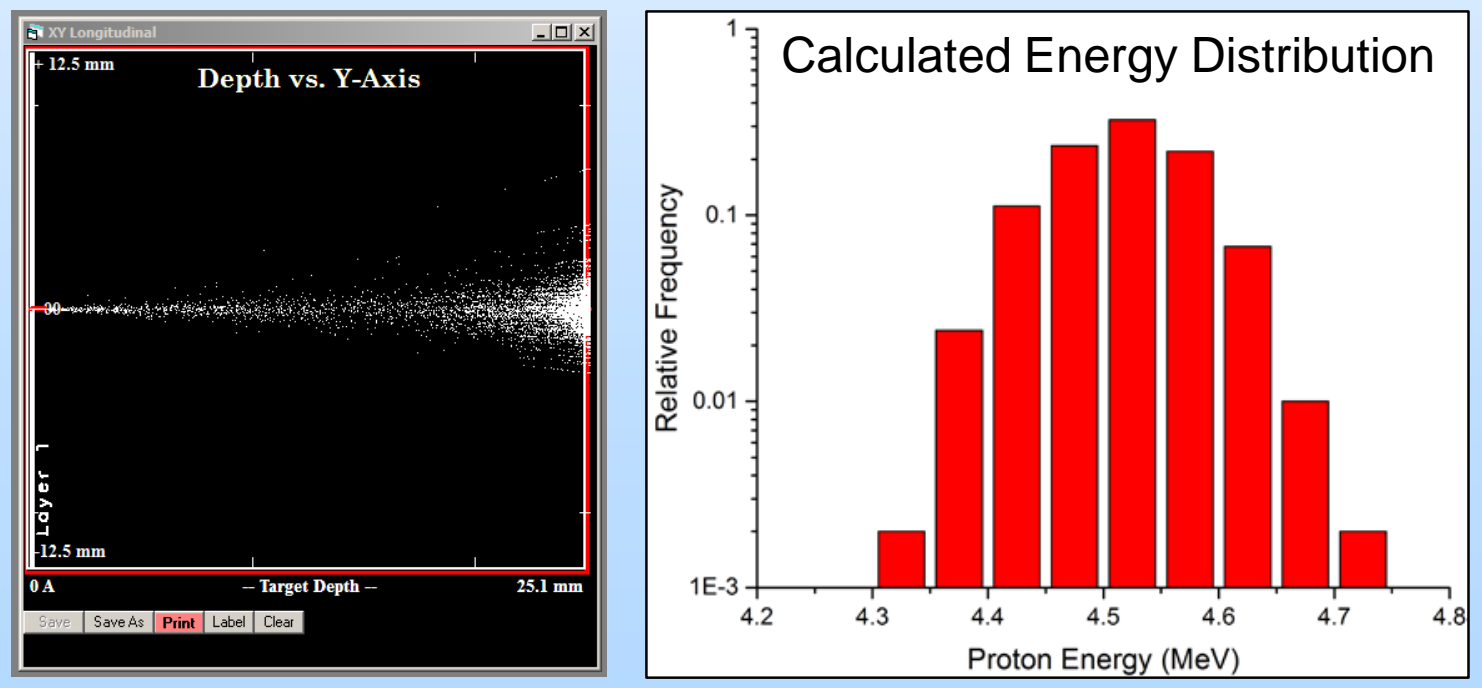


\section{Summary}

- Low-energy proton testing is challenging.

- At this point, there appears to be no suitable proxy for the observed single-event effects produced by low-energy protons.

- While alpha particle and proton SBU behavior seems to be proportional to LET, the MCU behavior is not.

- There are subsequent implications for radiation hardness assurance.

- May be more room for compromise at CMOS technology nodes $>65 \mathrm{~nm}$.

- Additional analysis techniques presented to aid data reduction efforts.

- Many technologies are flip-chip (such as our SRAM DUT), and die thickness uncertainty has always been one of the larger sources of systematic error. 


\section{Acknowledgements}

- NASA Electronic Parts and Packaging Program

- Defense Threat Reduction Agency

- National Reconnaissance Office

\section{Questions?}

